\title{
INFINITE SYSTEMS OF NONLINEAR OSCILLATION EQUATIONS RELATED TO THE STRING ${ }^{1}$
}

R. W. DICKEY

1. Introduction. The purpose of this paper is to discuss the existence and uniqueness of solutions to an infinite system of nonlinear oscillation equations of the form

$$
T_{j}^{\prime \prime}+j^{2}\left(a_{0}+a_{1} \sum_{l=1}^{\infty} l^{2} T_{l}^{2}\right) T_{j}=0, \quad j=1,2, \cdots, \infty,
$$

where the constants $a_{0}$ and $a_{1}$ satisfy the conditions $a_{0} \geqq 0$ and $a_{1}>0$ (the prime in (1.1) indicates differentiation with respect to $t$ ). The initial conditions on (1.1) will be taken as

$$
\begin{aligned}
& T_{j}(0)=\alpha_{j}, \\
& T_{j}^{\prime}(0)=\beta_{j} .
\end{aligned}
$$

Equations of the type (1.1) are related to the Duffing equation (cf. [1]), and arise in attempting to find Fourier series solutions

$$
W(x, t)=\sum_{j=1}^{\infty} T_{j}(t) \sin j \pi x / L,
$$

to the nonlinear partial integro-differential equation

$$
W_{t t}-\left(H_{0}+H_{1} \int_{0}^{L} W_{\xi}(\xi, t)^{2} d \xi\right) W_{x x}=0,
$$

$\left(H_{0} \geqq 0, H_{1}>0\right)$. Equation (1.4) describes the small amplitude vibrations of a string in which the dependence of the tension on the deformation cannot be neglected (cf. [2], [3]).

The equations (1.1) form an infinite Hamiltonian system, and in fact there is no difficulty in showing that any solution of (1.1) satisfies the condition

$$
\frac{d}{d t}\left(\sum_{j=1}^{\infty}\left(T_{j}^{\prime}\right)^{2}+a_{0} \sum_{j=1}^{\infty} j^{2} T_{j}^{2}+\frac{a_{1}}{2}\left(\sum_{j=1}^{\infty} j^{2} T_{j}^{2}\right)^{2}\right)=0 .
$$

Received by the editors May 12, 1969.

1 This research was sponsored by the National Science Foundation, Contract No. GP-7543. 
At first glance it would appear that if the initial conditions (1.2) satisfy a finite energy condition, i.e.,

$$
h=\sum_{j=1}^{\infty} \beta_{j}^{2}+a_{0} \sum_{j=1}^{\infty} j^{2} \alpha_{j}^{2}+\frac{a_{1}}{2}\left(\sum_{j=1}^{\infty} j^{2} \alpha_{j}^{2}\right)^{2}<\infty,
$$

then (1.1) should have a solution for all $t>0$. Indeed this is the case for finite systems of the form (1.1) since the finite system

$$
T_{j}^{\prime \prime}+j^{2}\left(a_{0}+a_{1} \sum_{l=1}^{N} l^{2} T_{l}^{2}\right) T_{j}=0, \quad j=1,2, \cdots, N,
$$

has associated with it a Lipschitz constant (depending on $N$ ). Thus the method of successive approximation (cf. [4]) may be used to show the existence of a solution to (1.7) locally, and the continuation of this solution is guaranteed by the fact that the energy-and hence the solution and its derivative-remains bounded. However, the infinite system of equations (1.1) is not Lipschitz continuous because of the unbounded nature of the coefficient of $T_{j}$ as $j \rightarrow \infty$. Thus the method of successive approximation fails and an alternative procedure is necessary.

In $\S 2$ of this paper, it will be shown that under certain conditions on the initial data (1.2), solutions of the finite system (1.7) converge to a solution of (1.1) as $N \rightarrow \infty$. In order to guarantee this it will be necessary to require that the initial data (1.2) satisfies a condition stronger than the simple finite energy condition (1.6). In $\$ 3$ it will be shown that the solution of (1.1) satisfying the initial conditions (1.2) is unique among a certain class of functions.

2. Existence. In proving the existence of solutions to (1.1), it is convenient to define a set of functions $T_{j, N}$ as follows: for $j \leqq N, T_{j, N}$ is to be a solution of the finite system of equations (1.7) and satisfy the initial conditions (1.2) for $j=1,2, \cdots, N$, and for $j>N$ set $T_{j, N} \equiv 0$. The functions $T_{j, N}$ are of course also solutions of the infinite system (1.1), i.e.

$$
T_{j, N}^{\prime \prime}+j^{2} A_{N} T_{j, N}=0, \quad j=1,2, \cdots, \infty,
$$

where

$$
A_{N}=a_{0}+a_{1} \sum_{l=1}^{\infty} l^{2} T_{l, N}^{2}
$$

If in addition the initial data (1.2) satisfies the finite energy condition (1.6) it follows that (cf. (1.5)) 


$$
\sum_{j=1}^{\infty}\left(T_{j, N}^{\prime}\right)^{2}+a_{0} \sum_{j=1}^{\infty} j^{2} T_{j, N}^{2}+\frac{a_{1}}{2}\left(\sum_{j=1}^{\infty} j^{2} T_{j, N}^{2}\right)^{2} \leqq h .
$$

Thus there exist constants $M_{1}$ and $M_{2}$ independent of $N$ such that

$$
\begin{gathered}
\sum_{j=1}^{\infty}\left(T_{j, N}^{\prime}\right)^{2} \leqq M_{1}, \\
\sum_{j=1}^{\infty} j^{2} T_{j, N}^{2} \leqq M_{2} .
\end{gathered}
$$

It is a consequence of (2.4b) that the functions $A_{N}$ are uniformly bounded independent of $N$. If it could also be shown that $\left|A_{N}^{\prime}\right|$ was uniformly bounded independent of $N$-so that the sequence $\left\{A_{N}\right\}$ is not only bounded but equicontinuous - the existence of a uniformly convergent subsequence would follow from the Arzela lemma (cf. [4]). Indeed the demonstration of the uniform boundedness of $\left|A_{N}^{\prime}\right|$ is the key step in proving the existence of solutions to (1.1). In what follows it will be necessary to assume that the initial data satisfies the conditions

$$
\begin{aligned}
& \sum_{j=1}^{\infty} j^{2} \beta_{j}^{2}<\infty, \\
& \sum_{j=1}^{\infty} j^{4} \alpha_{j}^{2}<\infty .
\end{aligned}
$$

This requirement on the initial data is, of course, stronger than the energy condition (1.6).

Lемма 2.1. If the initial data (1.2) satisfies the condition (2.5), and

$$
a_{0}+a_{1} \sum_{j=1}^{\infty} j^{2} \alpha_{j}^{2} \neq 0,
$$

there exists an interval $0 \leqq t<t_{c}$, such that $\left|A_{N}^{\prime}\right|$ is uniformly bounded independent of $N$ on any closed subinterval $0 \leqq t \leqq t^{*}<t_{c}$.

Proof. After differentiating the function $A_{N}$, the Schwarz inequality yields

$$
\begin{aligned}
\left|A_{N}^{\prime}\right| & \leqq 2 a_{1} \sum_{l=1}^{\infty} l^{2}\left|T_{l, N}\right|\left|T_{l, N}^{\prime}\right| \\
& \leqq 2 a_{1}\left\{\sum_{l=1}^{\infty} l^{2} T_{l, N}^{2} \sum_{l=1}^{\infty} l^{2}\left(T_{l, N}^{\prime}\right)^{2}\right\}^{1 / 2} \\
& \leqq 2\left\{a_{1} A_{N} \sum_{l=1}^{\infty} l^{2}\left(T_{l, N}^{\prime}\right)^{2}\right\}^{1 / 2} .
\end{aligned}
$$


Thus the object is to estimate the functions $\left|T_{j, N}^{\prime}\right|$. For this purpose define a function $E_{j, N}$ as (cf. [5])

$$
E_{j, N}=\frac{\left(T_{j, N}^{\prime}\right)^{2}}{j^{2} A_{N}}+T_{j, N}^{2} \geqq 0 .
$$

The condition (2.6) guarantees that $E_{j, N}$ is defined in some neighborhood of $t=0$ when $N$ is sufficiently large (the condition (2.6) is equivalent to the requirement that the partial differential equation (1.4) be hyperbolic at $t=0)$. The functions $T_{j, N}$ are solutions of $(2.1)$; therefore differentiation of (2.8) yields

$$
E_{j, N}^{\prime}=-\frac{A_{N}^{\prime}}{A_{N}}\left(\frac{\left(T_{j, N}^{\prime}\right)^{2}}{j^{2} A_{N}}\right) \leqq \frac{\left|A_{N}^{\prime}\right|}{A_{N}} E_{j, N}
$$

or equivalently

$$
E_{j, N} \leqq E_{j, N}(0) \exp \left(\int_{0}^{t} \frac{\left|A_{N}^{\prime}\right|}{A_{N}} d \tau\right) .
$$

Estimates on both $T_{j, v}$ and $T_{j, N}^{\prime}$ follow from (2.10). Thus it is found that

$$
\begin{aligned}
& T_{j, N}^{2} \leqq\left(\beta_{j}^{2} / j^{2} A_{N}(0)+\alpha_{j}^{2}\right) \exp \left(\int_{0}^{t} \frac{\left|A_{N}^{\prime}\right|}{A_{N}} d \tau\right), \\
& \left(T_{j, N}^{\prime}\right)^{2} / j^{2} A_{N} \leqq\left(\beta_{j}^{2} / j^{2} A_{N}(0)+\alpha_{j}^{2}\right) \exp \left(\int_{0}^{t} \frac{\left|A_{N}^{\prime}\right|}{A_{N}} d \tau\right) .
\end{aligned}
$$

Define $K_{N}$ as

$$
K_{N}=\sum_{j=1}^{\infty}\left(j^{2} \beta_{j}^{2} / A_{N}(0)+j^{4} \alpha_{j}^{2}\right),
$$

and note that finiteness of (2.12) follows from (2.5). In addition, the fact that $A_{N}(0) \leqq A_{\mathrm{N}+1}(0)$ shows that

$$
K_{N+1} \leqq K_{N}
$$

Combining (2.11b) and (2.7) it follows that

$$
\left|A_{N}^{\prime}\right| \leqq 2\left\{a_{1} A_{N}^{2} K_{N} \exp \left(\int_{0}^{t} \frac{\left|A_{N}^{\prime}\right|}{A_{N}} d \tau\right)\right\}^{1 / 2}
$$


or

$$
-\frac{d}{d t} \exp \left(-\frac{1}{2} \int_{0}^{t} \frac{\left|A_{N}^{\prime}\right|}{A_{N}} d \tau\right) \leqq\left(a_{1} K_{N}\right)^{1 / 2} \leqq\left(a_{1} K_{M}\right)^{1 / 2}
$$

if $N \geqq M$. It is a consequence of (2.15) that

$$
\exp \left(\frac{1}{2} \int_{0}^{t} \frac{\left|A_{N}^{\prime}\right|}{A_{N}} d \tau\right) \leqq 1 /\left(1-\left(a_{1} K_{M}\right)^{1 / 2} t\right)
$$

for all $N \geqq M$ and all $t$ in the interval

$$
0 \leqq t<t_{M}=1 /\left(a_{1} K_{M}\right)^{1 / 2} .
$$

Combining (2.14) and (2.16), it is clear that when $N \geqq M$ and $t$ is in the interval (2.17), $\left|A_{N}^{\prime}\right|$ satisfies the bound

$$
\left|A_{N}^{\prime}\right| \leqq 2\left(a_{1} K_{M}\right)^{1 / 2} A_{N} /\left(1-\left(a_{1} K_{M}\right)^{1 / 2} t\right) \text {. }
$$

Since $A_{N}$ is uniformly bounded independent of $N$, (2.18) shows that $\left|A_{N}^{\prime}\right|$ is also uniformly bounded independent of $N$ in the interval (2.17). In fact, if $K$ is defined as

$$
K=\lim _{N \rightarrow \infty} K_{N}
$$

and $t_{c}$ is defined as

$$
t_{c}=1 /\left(a_{1} K\right)^{1 / 2}
$$

then $\left|A_{N}^{\prime}\right|$ will be uniformly bounded in any closed interval $0 \leqq t$ $\leqq t^{*}<t_{c}$. Q.E.D.

It is of interest to note that, at least in the case where $a_{0}>0$, the interval $0 \leqq t<t_{c}$ grows arbitrarily large as the initial data (1.2) approaches zero.

In view of the preceding remarks, Lemma 2.1 guarantees the existence of a subsequence $\left\{A_{N_{i}}\right\}$ which converges uniformly to a (continuous) function $A(t)$ on any closed subinterval $0 \leqq t \leqq t^{*}<t_{c}$. Let $T_{j}$ be the solution of the (linear) equation

$$
T_{j}^{\prime \prime}+j^{2} A(t) T_{j}=0,
$$

satisfying the initial conditions (1.2). There is no difficulty in showing that $T_{j, N_{i}} \rightarrow T_{j}$ and $T_{j, N_{i}}^{\prime} \rightarrow T_{j}^{\prime}$ on the interval $0 \leqq t \leqq t^{*}<t_{c}$. The existence of solutions to (1.1) is settled by the following

THeOREM 2.1. The infinite system of equations (1.1) have a solution satisfying the initial data (1.2) on any closed interval $0 \leqq t \leqq t^{*}<t_{c}$ if the initial data satisfies the conditions (2.5) and (2.6). 
Proof. It is only necessary to show that the solutions of the linear system (2.21) furnish a solution of the system (1.1). For this purpose it suffices to show that

$$
A(t)=a_{0}+a_{1} \sum_{l=1}^{\infty} l^{2} T_{l}^{2}
$$

The series which occurs in (2.22) converges since (cf. (2.11) and (2.16))

$$
\begin{aligned}
T_{l}^{2} & \left.=\lim _{N_{i} \rightarrow \infty} T_{l, N_{i}}^{2} \leqq\left(\beta_{l}^{2} / l^{2} A(0)+\alpha_{l}^{2}\right) /\left(1-a_{1} K_{M}\right)^{1 / 2} t\right) \\
\left(T_{l}^{\prime}\right)^{2} & =\lim _{N_{i} \rightarrow \infty}\left(T_{l, N_{i}}^{\prime}\right)^{2} \leqq A\left(\beta_{l}^{2} / A(0)+l^{2} \alpha_{l}^{2}\right) /\left(1-\left(a_{1} K_{M}\right)^{1 / 2} t\right),
\end{aligned}
$$

for arbitrary $M$, and thus the series in (2.22) is majorized by a convergent series. The equality (2.22) follows from the estimate

$$
\begin{aligned}
& \left|A-a_{0}-a_{1} \sum_{l=1}^{\infty} l^{2} T_{l}^{2}\right| \leqq\left|A-A_{N_{i}}\right| \\
& \quad+a_{1} \sum_{l=1}^{n} l^{2}\left|T_{l}^{2}-T_{l, N_{i}}^{2}\right|+a_{1} \sum_{l=n+1}^{\infty} l^{2}\left(T_{l}^{2}+T_{l, N_{i}}^{2}\right) .
\end{aligned}
$$

The right side of (2.24) can be made arbitrarily small by first choosing $n$, then choosing $N_{i}$. Q.E.D.

The solution of (1.1) which has been constructed above has the properties

$$
\begin{aligned}
& \sum_{j=1}^{\infty} j^{4} T_{j}^{2}<\infty, \\
& \sum_{j=1}^{\infty} j^{2}\left(T_{j}^{\prime}\right)^{2}<\infty,
\end{aligned}
$$

and

$$
A(t)>0
$$

in the interval $0 \leqq t<t_{c}$. The convergence conditions (2.25) are an immediate consequence of (2.23) and (2.5). The condition (2.26) follows from the fact that if $A(\eta)=0$ for some value $t=\eta$ in the interval $0 \leqq t<t_{c}$, both $a_{0}=0$ and $T_{j}(\eta)=0$ for all $j$ (cf. (2.22)). If $T_{j}(\eta)=0$ for all $j$ the energy identity (1.5) shows that there exists at least one value of $j$ such that $T_{j}^{\prime}(\eta) \neq 0$. For this value of $j$, the inequality (2.23b) is violated at $t=\eta$. This contradiction proves (2.6). 
3. Uniqueness. In this section it will be shown that the infinite system (1.1) has at most one solution satisfying the initial conditions (1.2) and the conditions (2.25a) and (2.26).

Assume $T_{j}$ is a solution of (1.1) satisfying (2.25a) and (2.26) in some interval $0 \leqq t<\rho$. The function $A$ (cf. (2.22)) is differentiable in the interval $0 \leqq t<\rho$ since the Schwarz inequality implies

$$
\left|A^{\prime}\right| \leqq 2 a_{1}\left\{\sum_{l=1}^{\infty} l^{4} T_{l}^{2} \sum_{l=1}^{\infty}\left(T_{l}^{\prime}\right)^{2}\right\}^{1 / 2} .
$$

In view of (1.5) and (2.25a), both of the sums in (3.1) converge and are, in fact, uniformiy bounded on any closed subinterval $0 \leqq t$ $\leqq \rho^{*}<\rho$. In addition, the assumption that $A(t)>0$ for $0 \leqq t<\rho$ implies that there exists a constant $M$ such that

$$
\exp \left(\frac{1}{2} \int_{0}^{t} \frac{\left|A^{\prime}\right|}{A} d \tau\right) \leqq M
$$

for $0 \leqq t \leqq \rho^{*}<\rho$.

Let $T_{j}$ and $S_{j}$ be solutions of (1.1) satisfying the initial conditions (1.2) and the conditions (2.25a) and (2.26) for $0 \leqq t<\rho$, i.e. $T_{j}$ is a solution of (2.21) where $A$ is given by (2.22) and $S_{j}$ is a solution of

$$
S_{j}^{\prime \prime}+j^{2} B S_{j}=0
$$

where

$$
B=a_{0}+a_{1} \sum_{l=1}^{\infty} l^{2} S_{l}^{2}
$$

The difference

$$
U_{j}=T_{j}-S_{j}
$$

will be a solution of

$$
U_{j}^{\prime \prime}+j^{2} A U_{j}=j^{2}(B-A) S_{j},
$$

and satisfy the initial conditions

$$
U_{j}(0)=U_{j}^{\prime}(0)=0 .
$$

The object is to show that the only solution of (3.6) satisfying (3.7) is the trivial solution. If it could be shown that $U_{j}$, or some positive definite form involving $U_{j}$, satisfied a Gronwall inequality (cf. [6]) the result would follow. However, due to the form of (3.6), it is not clear that there exists such an inequality for $U_{j}$, and thus a different approach is necessary. 
It is convenient to begin by finding bounds on the solutions of (3.6). For this purpose define a function

$$
E_{j}=\left(U_{j}^{\prime}\right)^{2} / j^{2} A+U_{j}^{2} \geqq 0 .
$$

After differentiating (3.8), the differential equation (3.6) yields

$$
\begin{aligned}
E_{j}^{\prime} & =-\frac{A^{\prime}}{A}\left(\frac{\left(U_{j}^{\prime}\right)^{2}}{j^{2} A}\right)+2 \frac{(B-A)}{A} U_{j}^{\prime} S_{j} \\
& \leqq \frac{\left|A^{\prime}\right|}{A} E_{j}+2 \frac{|B-A|}{A}\left|U_{j}^{\prime}\right|\left|S_{j}\right|,
\end{aligned}
$$

or equivalently

$$
E_{j} \leqq 2 \exp \left(\int_{0}^{t} \frac{\left|A^{\prime}\right|}{A} d \tau\right) \int_{0}^{t} \frac{|B-A|}{A}\left|U_{j}^{\prime}\right|\left|S_{j}\right| d \tau .
$$

After summing over $j(3.10)$ becomes

$$
\sum_{j=1}^{\infty} E_{j} \leqq 2 \exp \left(\int_{0}^{t} \frac{\left|A^{\prime}\right|}{A} d \tau\right) \int_{0}^{t} I(\tau) d \tau
$$

where

$$
I(t)=\frac{|B-A|}{A} \sum_{j=1}^{\infty}\left|U_{j}^{\prime}\right|\left|S_{j}\right| .
$$

The function $I(t)$ may be estimated using the Schwarz inequality. Thus

$$
\begin{aligned}
|B-A| & \leqq a_{1} \sum_{j=1}^{\infty} j^{2}\left|S_{j}+T_{j}\right|\left|U_{j}\right| \\
& \leqq a_{1}\left\{\sum_{j=1}^{\infty} j^{4}\left(S_{j}+T_{j}\right)^{2} \sum_{j=1}^{\infty} U_{j}^{2}\right\}^{1 / 2}
\end{aligned}
$$

and

$$
\sum_{j=1}^{\infty}\left|U_{j}^{\prime}\right|\left|S_{j}\right| \leqq\left\{\sum_{j=1}^{\infty} j^{2} S_{j}^{2} \sum_{j=1}^{\infty}\left(U_{j}^{\prime}\right)^{2} / j^{2}\right\}^{1 / 2},
$$

so that

$$
I(t) \leqq G(t)\left\{\sum_{j=1}^{\infty} \frac{\left(U_{j}^{\prime}\right)^{2}}{j^{2} A} \sum_{j=1}^{\infty} U_{j}^{2}\right\}^{1 / 2}
$$


where

$$
G(t)=a_{1}\left\{\sum_{j=1}^{\infty} \frac{j^{2} S_{j}^{2}}{A} \sum_{j=1}^{\infty} j^{4}\left(S_{j}+T_{j}\right)^{2}\right\}^{1 / 2} .
$$

The important feature to observe about $G(t)$ is that it is bounded on any interval $0 \leqq t \leqq \rho^{*}<\rho$. Thus there exists a value of $t$, say $t=t_{1}$, such that

$$
t \exp \left(\int_{0}^{t} \frac{\left|A^{\prime}\right|}{A} d \tau\right) G(t)<1
$$

for all $t$ in the interval $0 \leqq t \leqq t_{1}$.

Lemma 3.1. $I(t) \equiv 0$ for $t$ in the interval $0 \leqq t \leqq t_{1}$.

Proof. Assume the maximum value of $I(t)$ in the interval $0 \leqq t \leqq t_{1}$ occurs at $t=\eta$. The inequality (3.11) implies that

$$
\sum_{j=1}^{\infty} E_{j}(\eta) \leqq 2 \eta \exp \left(\int_{0}^{\eta} \frac{\left|A^{\prime}\right|}{A} d \tau\right) I(\eta)
$$

or (cf. (3.15))

$$
\begin{aligned}
\sum_{j=1}^{\infty} E_{j}(\eta) \leqq & 2 \eta \\
& \cdot \exp \left(\int_{0}^{\eta} \frac{\left|A^{\prime}\right|}{A} d \tau\right) G(\eta)\left\{\sum_{j=1}^{\infty} \frac{\left(U_{j}^{\prime}\right)^{2}}{j^{2} A} \sum_{j=1}^{\infty} U_{j}^{2}\right\}_{t=\eta}^{1 / 2} .
\end{aligned}
$$

However, if $I(\eta) \neq 0,(3.17)$ and (3.19) imply that

$$
\sum_{j=1}^{\infty} E_{j}(\eta)<2\left\{\sum_{j=1}^{\infty} \frac{\left(U_{j}^{\prime}\right)^{2}}{j^{2} A} \sum_{j=1}^{\infty} U_{j}^{2}\right\}_{t=\eta}^{1 / 2}
$$

or, recalling the definition of $E_{j}$ (cf. (3.8)),

$$
\left\{\left(\sum_{j=1}^{\infty} \frac{\left(U_{j}^{\prime}\right)^{2}}{j^{2} A}\right)^{1 / 2}-\left(\sum_{j=1}^{\infty} U_{j}^{2}\right)^{1 / 2}\right\}_{t=\eta}^{2}<0 .
$$

This contradiction shows that $I(\eta)=0$, and hence $I(t) \equiv 0$ for $0 \leqq t$ $\leqq t_{1}$. Q.E.D.

The fact that $I(t) \equiv 0$ in the interval $0 \leqq t \leqq t_{1}$ combined with (3.11) shows that $U_{j}(t) \equiv 0$ and $U_{j}^{\prime}(t) \equiv 0$ in the interval. However, this result is easily extended to the interval $0 \leqq t<\rho$. Assume there exists some value of $j$ such that $U_{j}(t) \not \equiv 0$ for $0 \leqq t<\rho$. Let $\eta_{j}$ be the greatest lower bound of points for which $U_{j} \neq 0$ and let $\eta=$ g.l.b. $\eta_{j} \geqq t_{1}$. Since 
the functions $U_{j}$ are continuously differentiable for $0 \leqq t<\rho$ it follows that $U_{j}(\eta)=U_{j}^{\prime}(\eta)=0$. Since $\eta<\rho$ the development of this section may be repeated (with $t=\eta$ as the initial point) to show that $U_{j}$ and $U_{j}^{\prime}$ vanish in some interval to the right of $t=\eta$. Thus $\eta$ could not be the greatest lower bound of points for which $U_{j}$ does not vanish. This contradiction proves

THEOREM 3.1. The system of equations (1.1) have at most one solution satisfying the initial conditions (1.2) and the conditions (2.25a) and (2.26).

The solution of (1.1) which was constructed in $\$ 2$ satisfies the conditions (2.25a) and (2.26). It follows that if the initial data (1.2) satisfies (2.5) and (2.6), the system (1.1) has, on the interval $0 \leqq t<t_{c}$, exactly one solution satisfying (2.25a) and (2.26), and this solution is the limit of solutions to the finite system (1.7). The conditions (2.25a) and (2.26) may be interpreted in terms of the partial differential equation (1.4) and its solution (1.3). The condition (2.26) is simply the condition that (1.4) remain hyperbolic and (2.25a) is related to the convergence of the Fourier series (1.3). Indeed (2.25a) is essentially the condition that the Fourier series (1.3) be twice differentiable with respect to $x$ and $t$. In addition, the condition (2.5) and (2.6) are related to the differentiability of the initial conditions $W(x, 0)$ and $W_{t}(x, 0)$ in the partial differential equation (1.4). Thus, if the initial conditions $W(x, 0)$ and $W_{t}(x, 0)$ are sufficiently differentiable, the above discussion proves the existence of a solution to (1.4) of the form (1.3) in an interval $0 \leqq t<t_{c}$. Note also that if this solution does cease to exist for some value of $t \geqq t_{c}$ the cause will not be the unbounded growth of the solution (cf. (1.5)), but rather that it ceases to be sufficiently differentiable.

\section{REFERENCES}

1. J. J. Stoker, Nonlinear vibrations in mechanical and electrical systems, Interscience, New York, 1950.

2. S. Woinowsky-Krieger, The effect of axial forces on the vibrations of hinged bars, J. Appl. Mech. 17 (1950), 35-36.

3. J. G. Eisley, Nonlinear vibrations of beams and rectangular plates, Z. Angew. Math. Phys. 15 (1964), 167-175.

4. E. A. Coddington and N. Levinson, Theory of ordinary differential equations, McGraw-Hill, New York, 1955.

5. J. S. W. Wong, Explicit bounds for solutions of certain second order nonlinear differential equations, J. Math. Anal. Appl. 17 (1967), 339-342.

6. F. Brauer and J. A. Nohel, Ordinary differential equations, Benjamin, New York, 1967.

The University of Wisconsin, Madison 